\title{
Valeur moyenne des fonctions de Piltz sur les entiers sans grand facteur premier
}

\author{
par
}

\author{
Hikma Smida (Tunis)
}

1. Introduction. Désignons par $P(n)$ le plus grand facteur premier de $n$, avec la convention $P(1)=1$, et par $f(n)$ une fonction multiplicative. Pour $x \geq 1, y>1$ nous posons :

$$
\begin{gathered}
S(x, y):=\{n \leq x: P(n) \leq y\}, \\
\psi_{f}(x, y):=\sum_{n \in S(x, y)} f(n), \quad u:=\log x / \log y .
\end{gathered}
$$

Plusieurs problèmes en théorie des nombres nécessitent la connaissance du comportement asymptotique de $\psi_{f}(x, y)$ (cf. par exemple [7], [9], [11]).

Le cas le plus important concerne la fonction $\psi(x, y):=|S(x, y)|$. Cette fonction a été étudiée de manière approfondie par plusieurs auteurs ([3], [13], [16], [21]). On pourra aussi se reférer à [24] pour les principaux résultats concernant cette fonction et pour une liste plus complète de références bibliographiques. Nous mentionnons cependant deux résultats concernant $\psi(x, y)$.

(a) La formule asymptotique de Hildebrand [13],

$$
\psi(x, y)=x \varrho(u)\left(1+O\left(\frac{\log (1+u)}{\log y}\right)\right),
$$

valable uniformément dans le domaine

$$
x \geq 3, \quad \exp \left(\left(\log _{2} x\right)^{5 / 3+\varepsilon}\right) \leq y \leq x .
$$

Nous désignons, ici, par $\varrho(u)$ la fonction de Dickman. Elle est définie comme l'unique solution de l'équation différentielle aux différences avec conditions initiales

$$
\begin{cases}u \varrho^{\prime}(u)=-\varrho(u-1) & (u>1), \\ \varrho(u)=1 & (0 \leq u \leq 1), \\ \varrho(u)=0 & (u<0) .\end{cases}
$$


(b) L'estimation de Saias [21],

$$
\psi(x, y)=\Lambda(x, y)\left(1+O_{\varepsilon}\left(L_{\varepsilon}(y)^{-1}\right)\right),
$$

valable dans le domaine $\left(\mathrm{H}_{\varepsilon}\right)$. Avec les notations

$$
\Lambda(x, y):= \begin{cases}x \int_{1}^{\infty} \varrho(u-v) d\left(\left[y^{v}\right] \cdot y^{-v}\right) & (x \notin \mathbb{N}), \\ \frac{1}{2}(\Lambda(x-0, y)+\Lambda(x+0, y)) & (x \in \mathbb{N}),\end{cases}
$$

et

$$
L_{\varepsilon}(y):=\exp \left((\log y)^{3 / 5-\varepsilon}\right) .
$$

Nous citons un autre cas intéressant, celui de la fonction

$$
M(x, y):=\sum_{n \in S(x, y)} \mu(n) .
$$

Améliorant les résultats de Alladi [1] et de Hildebrand [15], Tenenbaum [26] montre que l'on peut approcher $M(x, y)$ par une fonction à variations régulières, ce qui permet d'obtenir une majoration non triviale pour le rapport $|M(x, y)| / \psi(x, y)$. Il démontre, en particulier, que l'on a

$$
M(x, y)=x \int_{0}^{\infty} \omega(u-v) M\left(y^{v}\right) y^{-v} d v+O\left(\psi(x, y) L_{\varepsilon}(y)^{-1}\right),
$$

uniformémemt dans le domaine

$$
x \geq 3, \quad \exp \left((\log x)^{2 / 5+\varepsilon}\right) \leq y \leq x .
$$

Dans (1.5) nous utilisons la notation

$$
M(x):=\sum_{n \leq x} \mu(n),
$$

et nous désignons par $\omega(u)$ la fonction de Buchstab. Elle est la solution de l'équation différentielle aux différences avec conditions initiales

$$
\begin{cases}{[u \omega(u)]^{\prime}=\omega(u-1)} & (2<u<+\infty), \\ u \omega(u)=1 & (1 \leq u \leq 2), \\ \omega(u)=0 & (-\infty<u<1) .\end{cases}
$$

D'autres fonctions ont été étudiées comme $f(n)=\mu^{2}(n)$ ([18], [19]); $f(n)=z^{\Omega(n)}([2],[5],[15])$, où $\Omega(n)$ désigne le nombre de facteurs premiers de l'entier $n ; f(n)=\tau_{k}(n)$ ([28], [29]) où $\tau_{k}(n)$ désigne le nombre de solutions en entiers $n_{1}, \ldots, n_{k} \geq 1$ de l'équation $n=n_{1} \ldots n_{k}$.

Signalons toutefois que dans [29] Xuan estime $\sum_{n \in S(x, y)} \tau_{k}(n)$ lorsque $k$ est entier en utilisant une méthode élémentaire basée sur la relation $\tau_{k}=$ $\tau_{k-1} * 1$ et la formule asymptotique de Hildebrand (1.1). 
D'une manière générale, de Bruijn et van Lint [4] ont estimé $\psi_{f}(x, y)$ lorsque $f$ est une fonction multiplicative positive satisfaisant aux conditions suivantes pour une constante positive convenable $k$ :

$$
\sum_{n \leq x} f(n) \sim x(\log x)^{k-1} L(\log x) \quad(x \rightarrow+\infty),
$$

où $L$ désigne une fonction à oscillations lentes, et pour chaque $u>1$ fixé

$$
\sum_{y<p<y^{u}} f(p) / p \sim k \log u \quad(y \rightarrow+\infty) .
$$

Ils ont établi que

$$
\psi_{f}(x, y) \sim \Gamma(k) u^{1-k} \varrho_{k}(u) \sum_{n \leq x} f(n) \quad\left(x:=y^{u} \rightarrow+\infty\right),
$$

uniformément pour $u$ borné. La fonction $\varrho_{k}(u)$ qui apparait dans (1.9) est la solution continue sur $\mathbb{R}^{*}$ de l'équation différentielle aux différences

$$
\begin{cases}u \varrho_{k}^{\prime}(u)=(k-1) \varrho_{k}(u)-k \varrho_{k}(u-1) & (u>1), \\ \varrho_{k}(u)=u^{k-1} / \Gamma(k) & (0<u \leq 1), \\ \varrho_{k}(u)=0 & (u \leq 0) .\end{cases}
$$

Le comportement asymptotique de $\varrho_{k}(u)$ est aujourd'hui bien connu ([12], [14], [23]). Dans [23], l'auteur donne un développement asymptotique de la fonction $\varrho_{k}(u)(k>0)$. Nous utilisons à cette fin une technique basée sur la méthode classique du point selle. Cette technique, développée ces dernières années, a permis de résoudre plusieurs problèmes d'arithmétique et d'améliorer un certain nombre de résultats ([24], [25]). C'est par cette même technique que nous nous proposons d'estimer $\psi_{f}(x, y)$ lorsque $f$ répond aux conditions (1.18).

Soit $z$ un nombre complexe et $\zeta(s)$ la fonction de Riemann; on définit la fonction arithmetique $\tau_{z}(n)$ (fonction de Piltz) par l'identité

$$
\zeta(s)^{z}:=\sum_{n \geq 1} \tau_{z}(n) n^{-s} \quad(\operatorname{Re} s>1)
$$

et on pose

$$
S_{z}(x):=\sum_{n \leq x} \tau_{z}(n) \quad(x \geq 2) .
$$

Dans [22], Selberg donne une formule asymptotique pour la fonction $S_{z}(x)$, à savoir

$$
S_{z}(x)=\frac{x}{\Gamma(z)}(\log x)^{z-1}+O\left(x(\log x)^{z-2}\right),
$$

valable uniformément, pour $A>0$, dans le domaine $|z| \leq A, x \geq 2$. 
Du théorème de Selberg, on peut déduire une estimation de la fonction sommatoire de toute fonction multiplicative dont la série de Dirichlet se comporte, au voisinage de $s=1$, comme une puissance de $\zeta(s)$ (cf. [24], théorème II.5.5). On a le résultat suivant :

$$
\sum_{n \leq x} f(n)=C_{f} x(\log x)^{z-1}+O\left(x(\log x)^{z-2}\right),
$$

valable pour $|z| \leq A, x \geq 2$, où $f(n)$ est une fonction multiplicative répondant aux conditions

$$
\left\{\begin{array}{l}
f=\tau_{z} * h, \\
\sum_{m \geq 1} \frac{h(m)}{m^{s}}(\log m)^{N} \text { converge absolument en } s=1 \\
\text { pour } N \leq[|z|]+3
\end{array}\right.
$$

avec

$$
C_{f}:=\sum_{m \geq 1} h(m) / m .
$$

Le but de ce travail est d'estimer $S_{k}(x, y):=\sum_{n \in S(x, y)} \tau_{k}(n)$, lorsque $k$ est un réel strictement positif fixé. Nous en déduirons une évaluation de $\psi_{f}(x, y)$ dans le cas où $f$ est une fonction multiplicative satisfaisant à certaines conditions que nous expliciterons plus loin. Nous étendrons ainsi le champ de validité du résultat de Xuan [29].

Pour tout réel $v \neq 1$, on définit $\xi(v)$ comme l'unique solution non nulle de l'équation

$$
e^{\xi(v)}=1+v \xi(v)
$$

avec la convention $\xi(1)=0$.

La fonction $\varrho_{k}(u)$ est définie par (1.10) et on désigne par $z_{k}(u)$ la solution de l'équation différentielle aux différences

$$
\begin{cases}u z_{k}^{\prime}(u)=-k z_{k}(u-1) & (u>1) \\ z_{k}(u)=1 & (0 \leq u \leq 1) \\ z_{k}(u)=0 & (u<0)\end{cases}
$$

On verra qu'en fait $\varrho_{k}(u)$ est la primitive fractionnaire d'ordre $k-1$ de la distribution $\delta_{0}+z_{k}$, où $\delta_{0}$ est la mesure de Dirac en zéro.

Le théorème 1 donne une estimation asymptotique de $S_{k}(x, y)$ qui généralise (1.1).

Théorème 1. Soient $\varepsilon>0, k>0$. Posons

$$
E_{k}(x, y):=\frac{1+|\xi(u)|}{\log y}+\frac{1}{(\log y)^{k}} .
$$


On a

$$
S_{k}(x, y)=x(\log y)^{k-1} \varrho_{k}(u)\left(1+O\left(E_{k}(x, y)\right)\right),
$$

uniformément dans le domaine

$$
x \geq 3, \quad \exp \left(\left(\log _{2} x\right)^{5 / 3+\varepsilon}\right) \leq y \leq x .
$$

Le théorème 2 est une estimation du type (1.3) et (1.5).

THÉORÈme 2. Soient $\varepsilon>0, k>0$. On a

$$
S_{k}(x, y)=x \int_{0}^{\infty} z_{k}(u-v) d\left(S_{k}\left(y^{v}\right) y^{-v}\right)\left(1+O\left(L_{\varepsilon}(y)^{-1}\right)\right),
$$

uniformément dans le domaine

$$
x \geq 3, \quad \exp \left((\log x)^{2 / 5+\varepsilon}\right) \leq y \leq x .
$$

Nous étendons ainsi le champ de validité d'un résultat de Hazlewood [10], tout en améliorant le terme d'erreur.

Enfin, le théorème 3 traite le cas d'une fonction multiplicative vérifiant

$$
\left\{\begin{array}{l}
f=\tau_{k} * h, \\
\sum_{\substack{m>t \\
P(m) \leq y}} \frac{|h(m)|}{m} \ll R_{\varepsilon}(t, y):=L_{\varepsilon / 2}(t)^{-1} \\
\quad+\exp \left(-\frac{\log t}{(\log y)^{2 / 5+\varepsilon / 2}}\right) \quad(t \geq 2, y \geq 2) .
\end{array}\right.
$$

ThÉorème 3. Soient $\varepsilon>0, k>0$. Pour $f$ vérifiant (1.18) et avec la notation (1.14), on a

$$
\psi_{f}(x, y)=C_{f} x(\log y)^{k-1} \varrho_{k}(u)\left(1+O\left(E_{k}(x, y)\right)\right),
$$

uniformément dans le domaine $\left(\mathrm{H}_{\varepsilon}\right)$.

L'auteur remercie G. Tenenbaum pour l'aide qu'il lui a apportée dans l'élaboration de ce travail.

2. Notations. Dans ce qui suit nous utilisons les notations suivantes.

La lettre $k$ dénote un réel strictement positif.

On désigne par $s$ un nombre complexe, les réels $\sigma$ et $t$ étant implicitement définis par

$$
s=\sigma+i t .
$$

Pour $s \in \mathbb{C} \backslash[0,+\infty[$, on note $\log s$ la valeur principale du logarithme en $s$. 
Pour $y>1$, on pose

$$
\zeta(s, y)^{k}:=\prod_{p \leq y}\left(1-p^{-s}\right)^{-k}, \quad \varphi_{1}(s, y):=\frac{d}{d s} \log \zeta(s, y) .
$$

Pour $v \geq 1$, on note $\xi(v)$ l'unique solution de l'équation

$$
e^{\xi(v)}-1=v \xi(v)
$$

avec la convention $\xi(1)=0$, et, lorsqu'aucune confusion n'est à craindre, on pose

$$
\xi:=\xi(u / k)
$$

On pose, pour tout nombre complexe $s$,

$$
I(s):=\int_{0}^{s} \frac{e^{v}-1}{v} d v
$$

avec la notation

$$
\sigma_{j}:=k I^{(j)}(\xi) \quad(j \geq 0) .
$$

La fonction $\widehat{f}(s)$ désigne la transformée de Laplace de la fonction $f$, soit

$$
\widehat{f}(s):=\int_{0}^{\infty} e^{-s t} f(t) d t .
$$

Le réel $\alpha$ est défini par

$$
\alpha:=1-\xi / \log y .
$$

Enfin, on pose pour tout $\varepsilon>0$

$$
L_{\varepsilon}(y):=\exp \left((\log y)^{3 / 5-\varepsilon}\right) \quad(y \geq 1) .
$$

3. Lemmes. Nous établissons tout d'abord certains résultats qui nous serons utiles par la suite.

Lemme 3.1. (i) Soient $\varepsilon>0, k>0$. Pour $y \geq y_{0}(\varepsilon, k), \sigma \geq 1-$ $(\log y)^{-2 / 5-\varepsilon},|t| \leq L_{\varepsilon}(y)$, on $a$

(3.1) $\zeta(s, y)^{k}=(\log y)^{k}((s-1) \zeta(s))^{k} \widehat{\varrho}_{k}((s-1) \log y)\left(1+O\left(L_{\varepsilon}(y)^{-1}\right)\right)$.

(ii) En particulier, pour $\alpha=1-\xi / \log y, 1 \leq u \leq L_{\varepsilon}(y)$, on a

$$
\zeta(\alpha, y)^{k}=(\log y)^{k} e^{k \gamma+\sigma_{0}}\left(1+O\left(\frac{1+|\xi|}{\log y}\right)\right) .
$$

Démonstration. (i) Nous savons d'après [12] que

$$
\widehat{\varrho}_{k}(s)=\exp (k \gamma+k I(-s)) \text {. }
$$

Puisque $\varrho_{1}=\varrho$, on en déduit

$$
\widehat{\varrho}_{k}(s)=\widehat{\varrho}(s)^{k} .
$$


Le point (i) est une conséquence immédiate de (3.4) et du lemme III.5.9.1 de [24].

(ii) La condition $1 \leq u \leq L_{\varepsilon}(y)$ nous permet d'appliquer (3.1) avec $s=\alpha$. Il vient

$$
\zeta(\alpha, y)^{k}=(\log y)^{k}((\alpha-1) \zeta(\alpha))^{k} \widehat{\varrho}_{k}((\alpha-1) \log y)\left(1+O\left(L_{\varepsilon}(y)^{-1}\right)\right) .
$$

Or

$$
(\alpha-1) \zeta(\alpha)=1+O(|\alpha-1|)=1+O(|\xi| / \log y) .
$$

La formule (3.2) en découle, compte tenu de (3.3), $\sigma_{0}$ étant donné par (2.1) avec $j=0$.

Le lemme suivant, prouvé par Fouvry et Tenenbaum (cf. le lemme 6.1 de [8]) permet une estimation de $\widehat{\varrho}_{k}(s)$.

Lemme 3.2. Pour $s \in \mathbb{C} \backslash]-\infty, 0], k>0$, on $a$

$$
\widehat{\varrho}_{k}(s)=\frac{1}{s^{k}} \exp (-k \mathfrak{J}(s)) \text {, }
$$

où nous avons posé

$$
\mathfrak{J}(s):=\int_{0}^{\infty} \frac{e^{-s-v}}{s+v} d v .
$$

On déduit de la formule (3.5) et de la majoration

$$
\mathfrak{J}(s) \ll e^{-\sigma}|t|^{-1}
$$

que

$$
\widehat{\varrho}_{k}(s)= \begin{cases}\frac{1}{s^{k}}\left(1+O\left(\frac{e^{-\sigma}}{|t|}\right)\right) & \left(|t|>e^{-\sigma}\right), \\ \frac{1}{(i t)^{k}}\left(1+O\left(\frac{e^{-\sigma}+|\sigma|}{|t|}\right)\right) & \left(|t|>\max \left(e^{-\sigma},|\sigma|\right)\right) .\end{cases}
$$

Nous rappelons enfin deux résultats prouvés dans [23] (lemmes 4.10, 4.11 et théorème 1).

Lemme 3.3. Pour $k>0, u>\max (1, k), s=-\xi+i t, t \in \mathbb{R}$, on $a$

$$
\widehat{\varrho}_{k}(s) \ll \begin{cases}\exp \left(k \gamma+\sigma_{0}-\frac{2 t^{2}}{\pi^{2}} \sigma_{2}\right) & (|t| \leq \pi), \\ \exp \left(k \gamma+\sigma_{0}-\frac{u}{\xi^{2}+\pi^{2}}\right) & (|t|>\pi) .\end{cases}
$$

Lemme 3.4. Soit $k$ un entier $\geq 0$ et $\varepsilon>0$. On a uniformément pour $u>1, k \geq \varepsilon, \xi=\xi(u / k)$

$$
\varrho_{k}(u)=\frac{e^{k \gamma+\sigma_{0}-u \xi}}{\sqrt{2 \pi \sigma_{2}}}\left(1+O_{\varepsilon}\left((u+k)^{-1}\right)\right) .
$$


Une formule asymptotique d'ordre arbitrairement grand étendant (3.8) est établie dans [23].

D'après le lemme 4.2 de [23], on a $\varrho_{k}(v)>0$ pour tout $v>0$. Il suit, compte tenu des estimations $\sigma_{0} \sim u$ et $\xi \sim \log (u / k)$, valables pour $u>k$ (cf. [23], lemmes 4.4 et 4.3 ), et de l'expression (3.8)

$$
\varrho_{k}(u) \gg u^{-2 u} \quad(u \geq 1) .
$$

Nous démontrons à présent quelques lemmes utiles à la preuve des théorèmes $1,2,3$.

Lemme 3.5. Pour $s=-\xi+i t, 1 \leq u \leq k$, on $a$

$$
\widehat{\varrho}_{k}(s)=O_{k}(1) \text {. }
$$

Démonstration. D'après le lemme 4.3 de [23] on a $-\xi \leq k / u \leq k$ pour $u \geq 1$, de plus $\varrho_{k}(v)>0$ pour $v>0$. Il suit pour $\sigma=-\xi$

$$
\left|\varrho_{k}(s)\right|=\left|\int_{0}^{\infty} \varrho_{k}(v) e^{-s v} d v\right| \leq \int_{0}^{\infty} \varrho_{k}(v) e^{k v} d v=\widehat{\varrho}_{k}(-k),
$$

d'où (3.10).

Lemme 3.6. Soient $\varepsilon>0$ et $k>0$. Pour $y \geq 2, \alpha=1-\xi / \log y$, $1<u \leq L_{\varepsilon}(y)$, on $a$

$$
\begin{aligned}
& x^{\alpha} \zeta(\alpha, y)^{k} \\
& \quad=x(\log y)^{k} \varrho_{k}(u)(2 \pi u)^{1 / 2}\left(1+O_{\varepsilon}\left(\frac{1+|\xi|}{\log y}+\frac{1}{\log (1+u)}\right)\right) .
\end{aligned}
$$

Démonstration. L'évaluation (3.11) découle facilement de (3.2), (3.8) et de l'estimation

$$
\sigma_{2}=u\left(1+O\left(\frac{1}{1+\log (u / k)}\right)\right) \quad(u>k),
$$

prouvée dans [23], lemme 4.5.

Lemme 3.7. Soient $\varepsilon>0$ et $k>0$. Pour $y \geq y_{0}(\varepsilon, k), 1 \leq u \leq L_{\varepsilon}(y)$, $T=L_{\varepsilon / 2}(y)$, on $a$

$$
x^{\alpha} \sum_{\substack{|\log (x / n)| \leq T^{-1 / 2} \\ P(n) \leq y}} \frac{\tau_{k}(n)}{n^{\alpha}} \ll_{\varepsilon, k} x \varrho_{k}(u) L_{\varepsilon}(y)^{-1} .
$$

Démonstration. La démonstration est essentiellement identique à celle du lemme III.5.9.4 de [24]. 
Désignons par $R_{T}$ le membre de gauche de (3.12). On considère en premier lieu le cas

$$
u \geq(\log y)^{3 / 5-2 \varepsilon / 3} .
$$

On introduit alors la fonction de poids

$$
\chi(t):=\left(\frac{\sin ((1 / 2) t \sqrt{T})}{(1 / 2) t \sqrt{T}}\right)^{2},
$$

dont la transformée de Fourier est donnée par

$$
\widehat{\chi}(v)=\frac{1}{\sqrt{T}} \max (1-|v| / \sqrt{T}, 0) .
$$

Il suit

$$
\begin{aligned}
R_{T} & \ll x^{\alpha} \sum_{\substack{n \geq 1 \\
P(n) \leq y}} \frac{\tau_{k}(n)}{n^{\alpha}} \chi(\log (x / n)) \\
& \ll \sum_{\substack{n \geq 1 \\
P(n) \leq y}} \tau_{k}(n)\left(\frac{x}{n}\right)^{\alpha} \int_{\mathbb{R}} \widehat{\chi}(v) e^{i v \sqrt{T} \log (x / n)} d v .
\end{aligned}
$$

Le changement de variable $w=v \sqrt{T}$ nous permet d'écrire

$$
R_{T} \ll \frac{1}{T} \int_{|w| \leq T}\left(1-\frac{|w|}{T}\right) \zeta(\alpha+i w, y)^{k} x^{\alpha+i w} d w \ll \frac{1}{T}\left(I_{1}+I_{2}\right),
$$

où $I_{1}$ et $I_{2}$ correspondent aux domaines d'intégration respectifs $|w| \leq T^{1 / 2}$, $T^{1 / 2} \leq|w| \leq T$.

On a clairement $I_{1} \ll x^{\alpha} \zeta(\alpha, y)^{k} T^{1 / 2}$, ce qui implique, compte tenu de (3.11), que

$$
I_{1} \ll x(\log y)^{k} \varrho_{k}(u) \sqrt{u} T^{1 / 2} \ll x \varrho_{k}(u) T^{3 / 4} .
$$

Estimons $I_{2}$. On a

$$
I_{2} \ll x^{\alpha} \max _{T^{1 / 2} \leq w \leq T}\left|\zeta(\alpha+i w, y)^{k}\right| T .
$$

En appliquant (3.1) avec $s=\alpha+i w$, on obtient

$$
\zeta(s, y)^{k} \ll \zeta(s)^{k}((s-1) \log y)^{k} \widehat{\varrho}_{k}((s-1) \log y) .
$$

Pour $w \geq T^{1 / 2}$, on a bien $|w \log y| \geq 1+u \xi$. Il suit grâce à (3.6)

$$
\zeta(s, y)^{k} \ll \zeta(s)^{k} \ll(\log T)^{k} .
$$

Ainsi on peut écrire

$$
I_{2} \ll x^{\alpha} T(\log T)^{k} \ll x e^{-u \xi} T(\log T)^{k} .
$$


Il résulte de (3.8), (3.13) et de l'estimation $\sigma_{0} \sim u$ (cf. lemme 4.4 de [23]) que

$$
I_{2} \ll x \varrho_{k}(u) L_{\varepsilon}(y)^{-1} T .
$$

En reportant (3.15) et (3.16) dans (3.14), on obtient

$$
R_{T} \ll x \varrho_{k}(u) L_{\varepsilon}(y)^{-1},
$$

ce qui est de l'ordre requis.

Considérons à présent le cas

$$
u<(\log y)^{3 / 5-2 \varepsilon / 3} .
$$

D'après l'inégalité de Cauchy-Schwarz on a

$$
R_{T} \ll \sqrt{\sum_{n \leq 2 x}\left(\tau_{k}(n)\right)^{2} \sum_{|\log (x / n)| \leq T^{-1 / 2}} 1 .}
$$

On déduit de (1.12) et de la majoration triviale $\sum_{|\log (x / n)| \leq T} 1 \ll x / T$ que

$$
R_{T} \ll x(\log x)^{\left(k^{2}-1\right) / 2} T^{-1 / 4} .
$$

Par ailleurs la fonction $\varrho_{k}(u)$ est strictement positive pour $u>0$. Il suit, en tenant compte de (3.9),

$$
R_{T} \ll x \varrho_{k}(u) u^{2 u} T^{-1 / 4}(\log x)^{\left(k^{2}-1\right) / 2} \ll x \varrho_{k}(u) L_{\varepsilon}(y)^{-1} .
$$

Le lemme est ainsi établi.

Lemme 3.8. Soit $z_{k}(u)$ la fonction définie par (1.15). Alors on a

(i) $z_{k}(u)$ est continue en tout point $u \neq 0$ et possède une discontinuité de première espèce en $u=0$. On peut la prolonger par continuité à droite sur $\mathbb{R}$. De plus, elle est dérivable en tout point $u \neq 0,1$.

(ii) $z_{k}^{\prime}(u)$ admet une discontinuité de première espèce en $u=1$ et on peut la prolonger par continuité à droite sur $\mathbb{R}$. Elle est dérivable en tout point $u \neq 0,1,2$; et la fonction $z_{k}^{\prime \prime}(u)$ possède une discontinuité de première espèce en $u=1,2$.

De plus on a

$$
\varrho_{k}(u)=\frac{1}{\Gamma(k)}\left(u^{k-1}+\int_{0}^{u} z_{k}^{\prime}(u-v) v^{k-1} d v\right) \quad(u>0) .
$$

Démonstration. Le point (i) découle aisément de (1.15).

Montrons (ii). On déduit de (1.15) que l'on a

$$
\begin{cases}u z_{k}^{\prime}(u)=-k z_{k}(u-1) & (u>0, u \neq 1) \\ z_{k}^{\prime}(u)=0 & (0<u<1)\end{cases}
$$


Il en résulte que $z_{k}^{\prime}(u)$ possède une discontinuité de première espèce en $u=1$, et on peut la prolonger par continuité à droite sur $\mathbb{R}$. De plus on peut écrire, d'après $(*)$, que $z_{k}^{\prime}(u)$ est dérivable pour tout $u \neq 0,1,2$ et que l'on a

$$
\begin{cases}u z_{k}^{\prime \prime}(u)=-k z_{k}^{\prime}(u-1)-z_{k}^{\prime}(u) & (u>1, u \neq 2), \\ z_{k}^{\prime \prime}(u)=0 & (0<u<1) .\end{cases}
$$

Il suit que $z_{k}^{\prime \prime}(u)$ possède une discontinuité de première espèce en $u=1,2$ et on peut la prolonger par continuité à droite.

Montrons (3.18). Lorsque $0<u<1$ on a $z_{k}^{\prime}(u-v)=0(0 \leq v<u)$, donc

$$
\frac{1}{\Gamma(k)}\left(u^{k-1}+\int_{0}^{u} z_{k}^{\prime}(u-v) v^{k-1} d v\right)=\frac{u^{k-1}}{\Gamma(k)}=\varrho_{k}(u) .
$$

Supposons $u>1$ et posons

$$
f(u):=u^{k-1}+\int_{0}^{u} z_{k}^{\prime}(u-v) v^{k-1} d v .
$$

Remarquons que l'intégrale est convergente pour $k>0$. De plus on a d'après (ii)

$$
f^{\prime}(u)=(k-1) u^{k-2}+\int_{0}^{u} z_{k}^{\prime \prime}(u-v) v^{k-1} d v-k(u-1)^{k-1} \quad(u>1),
$$

où le dernier terme provient de la discontinuité de $z_{k}^{\prime}(u)$ en $u=1$. En multipliant par $u$, on obtient

$$
\begin{aligned}
u f^{\prime}(u)=(k-1) u^{k-1}+\int_{0}^{u}(u-v) z_{k}^{\prime \prime}(u-v) v^{k-1} d v & \\
& +\int_{0}^{u} z_{k}^{\prime \prime}(u-v) v^{k} d v-k u(u-1)^{k-1} .
\end{aligned}
$$

Or, d'après $(*)$, on a

$$
\begin{aligned}
\int_{0}^{u} v^{k} d z_{k}^{\prime}(u-v) & =\left[v^{k} z_{k}^{\prime}(u-v)\right]_{0}^{u}-k \int_{0}^{u} z_{k}^{\prime}(u-v) v^{k-1} d v \\
& =-\int_{0}^{u} v^{k} z_{k}^{\prime \prime}(u-v) d v+k(u-1)^{k} .
\end{aligned}
$$

Il suit

$$
\begin{aligned}
u f^{\prime}(u)= & (k-1) u^{k-1}-\int_{0}^{u}\left(k z_{k}^{\prime}(u-1-v)+z_{k}^{\prime}(u-v)\right) v^{k-1} d v \\
& +k \int_{0}^{u} z_{k}^{\prime}(u-v) v^{k-1} d v+k(u-1)^{k}-k u(u-1)^{k-1},
\end{aligned}
$$


où l'on a tenu compte de $(* *)$. D'où

$$
\begin{aligned}
u f^{\prime}(u)= & (k-1) u^{k-1}+(k-1) \int_{0}^{u} z_{k}^{\prime}(u-v) v^{k-1} d v \\
& -k \int_{0}^{u} z_{k}^{\prime}(u-1-v) v^{k-1} d v-k(u-1)^{k-1} \\
= & (k-1) f(u)-k f(u-1) .
\end{aligned}
$$

Sachant que

$$
\Gamma(k) f(u)=\varrho_{k}(u) \quad(0<u \leq 1),
$$

on en déduit que cette relation persiste pour tout $u>0$, d'où (3.18).

Lemme 3.9. Pour $k>0, s=\sigma+i t, \sigma>0$, on $a$

$$
s^{k} \widehat{\varrho}_{k}(s)=s \widehat{z}_{k}(s)=1+\widehat{z}_{k}^{\prime}(s) .
$$

Démonstration. Posons

$$
g(v)= \begin{cases}v^{k-1} / \Gamma(k) & (v \geq 0), \\ 0 & (v<0) .\end{cases}
$$

Pour $k>0$, on a

$$
\widehat{g}(s)=s^{-k} .
$$

On déduit de la formule (3.18) et du lemme 3.8 que

$$
\varrho_{k}(u)=g(u)+\left(z_{k}^{\prime} * g\right)(u) \quad(u>0) .
$$

D'après le lemme 3 de [17], on a $z_{k}^{\prime}(u) \ll u^{c}$. Il suit que $\int_{0}^{\infty} e^{-s u} z_{k}^{\prime}(u) d u$ converge pour $\sigma>0$. Le théorème de convolution nous permet alors d'écrire

$$
\widehat{\varrho}_{k}(s)=\widehat{g}(s)\left(1+\widehat{z}_{k}^{\prime}(s)\right) \quad\left(\sigma \in \mathbb{R}_{+}^{*}\right) .
$$

Le lemme en découle, compte tenu de (3.20).

Avant d'énoncer le lemme suivant, nous introduisons quelques notations supplémentaires. On pose pour $\varepsilon>0$ fixé

$$
\vartheta:=\left\{s: \sigma(t)>1-c /(\log (1+|t|))^{(2+\varepsilon) / 3}\right\},
$$

où $c$ est une constante suffisamment petite pour que $\zeta(s)$ n'ait aucun zéro $\sigma+i t$ tel que $\sigma \geq \sigma(t)$ (cf. [6]);

$T:=L_{\varepsilon / 2}(y)$,

$T_{0}$ est l'intersection de la droite $\sigma=\alpha$ avec la courbe $\sigma=\sigma(t)$,

$\kappa:=1+1 / \log x$,

$T_{1}:=L_{\varepsilon / 3}(x)$,

$Z$ est le chemin symétrique par rapport à l'axe réel dont la partie supérieure est constituée des arcs $\Gamma_{1}, \Gamma_{2}, \Gamma_{3}, \Gamma_{4}$ définis respectivement par

$$
\Gamma_{1}:=\left\{\alpha+i t: T \leq t \leq T_{0}\right\},
$$


$\Gamma_{2}:=\left\{\sigma(t)+i t: T_{0} \leq t \leq T_{1}\right\}$,

$\Gamma_{3}:=\left\{\sigma+i T_{1}: \sigma\left(T_{1}\right) \leq \sigma \leq \kappa\right\}$,

$\Gamma_{4}:=\left\{\kappa+i t: T_{1} \leq t\right\}$.

Lemme 3.10. Soit $0<\varepsilon<1$. Pour $k>0, u \leq L_{\varepsilon}(y)$, on a

$$
\int_{Z} \zeta(s)^{k} \frac{x^{s}}{s} d s \ll x L_{\varepsilon / 2}(x)^{-1}+x \varrho_{k}(u) L_{\varepsilon}(y)^{-1} .
$$

En particulier, dans le domaine $\left(\mathrm{G}_{\varepsilon}\right)$, la majoration précédente est

$$
\ll x \varrho_{k}(u) L_{\varepsilon}(y)^{-1} .
$$

Démonstration. D'après la majoration classique (cf. [24], théorème II.3.7)

$$
\zeta(s)^{k} \ll(\log (1+|t|))^{k} \quad\left(\sigma \geq 1-c /(\log (1+|t|))^{(2+\varepsilon) / 3}\right),
$$

on a

$$
\int_{\Gamma_{1}} \zeta(s)^{k} \frac{x^{s}}{s} d s \ll x^{\alpha}\left(\log T_{0}\right)^{k+1} .
$$

Lorsque $u \geq(\log y)^{3 / 5-2 \varepsilon / 3}$, on a, compte tenu du lemme 3.4,

$$
x^{\alpha}(\log T)^{k+1} \ll x \varrho_{k}(u) L_{\varepsilon}(y)^{-1} .
$$

Lorsque $u<(\log y)^{3 / 5-2 \varepsilon / 3}$, on peut écrire

$$
\int_{\Gamma_{1}} \zeta(s)^{k} \frac{x^{s}}{s} d s=\int_{W} \zeta(s)^{k} \frac{x^{s}}{s} d s
$$

où $W$ est le chemin orienté formé des segments

$$
[\alpha+i T, \kappa+i T], \quad\left[\kappa+i T, \kappa+i T_{0}\right], \quad\left[\alpha+i T_{0}, \kappa+i T_{0}\right] .
$$

Il suit

$$
\int_{\Gamma_{1}} \zeta(s)^{k} \frac{x^{s}}{s} d s \ll x \frac{(\log T)^{k}}{T}+x^{\kappa} \sum_{n \geq 1} \frac{\tau_{k}(n)}{n^{\kappa}(1+T|\log (x / n)|)} .
$$

Il découle de (3.9) que le premier terme du membre de droite de l'estimation précédente est $\ll x \varrho_{k}(u) L_{\varepsilon}(y)^{-1}$.

On estime la somme de l'expression précédente comme dans le lemme III.5.9.4 de [24], en considérant séparément les cas $|x-n| \leq x T^{-1 / 4}$ et $|x-n|>x T^{-1 / 4}$. On a

$$
x^{\kappa} \sum_{|x-n|>x T^{-1 / 4}} \frac{\tau_{k}(n)}{n^{\kappa}(1+T|\log (x / n)|)} \ll x T^{-3 / 4} \ll x \varrho_{k}(u) L_{\varepsilon}(y)^{-1},
$$

où l'on a tenu compte de (3.9). 
De plus, on peut écrire grâce à l'inégalité de Cauchy-Schwarz

$$
\begin{aligned}
& x^{\kappa} \sum_{|x-n| \leq x T^{-1 / 4}} \frac{\tau_{k}(n)}{n^{\kappa}(1+T|\log (x / n)|)} \\
& \ll \sqrt{\sum_{n \leq 2 x}\left(\tau_{k}(n)\right)^{2} \sum_{|x-n| \leq x T^{-1 / 4}} 1} \\
& \ll x(\log x)^{\left(k^{2}-1\right) / 2} T^{-1 / 8} \ll x \varrho_{k}(u) L_{\varepsilon}(y)^{-1}
\end{aligned}
$$

d'après (3.9).

Ainsi on a bien

$$
\int_{\Gamma_{1}} \zeta(s)^{k} \frac{x^{s}}{s} d s \ll x \varrho_{k}(u) L_{\varepsilon}(y)^{-1} .
$$

L'intégrale sur $\Gamma_{1}$ est donc bien de l'ordre requis.

Estimons l'intégrale sur $\Gamma_{2}$. La fonction $\sigma(t)$ étant croissante, on peut écrire

$$
\int_{\Gamma_{2}} \zeta(s)^{k} \frac{x^{s}}{s} d s \ll x^{\sigma\left(T_{1}\right)}\left(\log T_{1}\right)^{k+1} \ll x L_{\varepsilon / 2}(x)^{-1} .
$$

On obtient similairement

$$
\int_{\Gamma_{3}} \zeta(s)^{k} \frac{x^{s}}{s} d s \ll x(\log x)^{k} L_{\varepsilon / 3}(x)^{-1} \ll x L_{\varepsilon / 2}(x)^{-1} .
$$

Enfin, pour estimer l'intégrale sur $\Gamma_{4}$, on écrit

$$
\int_{\Gamma_{4}} \zeta(s)^{k} \frac{x^{s}}{s} d s \ll x^{\kappa} \sum_{n \geq 1} \frac{\tau_{k}(n)}{n^{\kappa}\left(1+T_{1}|\log (x / n)|\right)} \ll x L_{\varepsilon / 2}(x)^{-1},
$$

où nous avons procédé comme pour la preuve de (3.23).

Le lemme est ainsi établi.

4. Démonstration du théorème 1. Nous établissons la proposition suivante. Nous verrons qu'associée aux lemmes 3.6 et 3.7, elle permet d'établir facilement le théorème 1 .

Proposition 4.1. Soit $0<\varepsilon<1$. Pour $k \in \mathbb{R}_{+}^{*}-\{1\}, y \geq y_{0}(\varepsilon, k)$, $s=\alpha+i t$, on $a$

$$
\frac{1}{2 i \pi} \int_{\alpha-i / 2}^{\alpha+i / 2} \zeta(s, y)^{k} \frac{x^{s}}{s} d s=x(\log y)^{k-1} \varrho_{k}(u)\left(1+O\left(E_{k}(x, y)\right)\right),
$$

uniformément dans le domaine $\left(\mathrm{H}_{\varepsilon}\right)$. 
Démonstration. Notons $K(u)$ le membre de gauche de (4.1), et posons

$$
F(s):=s^{-1}((s-1) \zeta(s))^{k}-1 .
$$

D'après le lemme 3.1 on a

$$
K(u)=\frac{1}{2 i \pi} \int_{\alpha-i / 2}^{\alpha+i / 2}(\log y)^{k} \widehat{\varrho}_{k}((s-1) \log y)\left(1+F(s)+O\left(\frac{\zeta(s, y)^{k}}{L_{\varepsilon / 2}(y)}\right)\right) x^{s} d s .
$$

On déduit aisément de (3.2) que la contribution du terme d'erreur à l'intégrale est

$$
\ll x^{\alpha} \zeta(\alpha, y)^{k} L_{\varepsilon / 2}(y)^{-1} \ll x \varrho_{k}(u) L_{\varepsilon}(y)^{-1},
$$

ce qui est bien de l'ordre requis. Ainsi, lorsqu'on effectue le changement de variable $(s-1) \log y=v:=\beta+i t$, on obtient

$$
K(u)=x(\log y)^{k-1}\left(K_{1}(u)+K_{2}(u)\right)+O\left(x \varrho_{k}(u) L_{\varepsilon}(y)^{-1}\right),
$$

avec

$$
\begin{aligned}
& K_{1}(u):=\frac{1}{2 i \pi} \int_{\substack{\beta=-\xi \\
|t| \leq(\log y) / 2}} \widehat{\varrho}_{k}(v) e^{u v} d v, \\
& K_{2}(u):=\frac{1}{2 i \pi} \int_{\substack{\beta=-\xi \\
|t| \leq(\log y) / 2}} \widehat{\varrho}_{k}(v) F(1+v / \log y) e^{u v} d v,
\end{aligned}
$$

$K_{1}(u)$ constitue le terme principal de (4.1).

Nous traiterons $K_{2}(u)$ comme un terme d'erreur.

Estimation de $K_{1}(u)$. La fonction $\varrho_{k}(u)$ est unimodale pour $k>1$ et strictement décroissante pour $k<1$. De plus l'intégrale $\int_{0}^{\infty} \varrho_{k}(v) e^{-s v} d v$ converge sur toute droite $s=\sigma+i t$. On peut donc appliquer le théorème d'inversion de Laplace et écrire

$$
K_{1}(u)=\frac{1}{2 i \pi} \int_{-\xi-i \infty}^{-\xi+i \infty} \widehat{\varrho}_{k}(v) e^{u v} d v-\frac{1}{2 i \pi} \int_{\substack{\beta=-\xi \\|t|>(\log y) / 2}} \widehat{\varrho}_{k}(v) e^{u v} d v,
$$

soit

$$
K_{1}(u)=\varrho_{k}(u)+K_{11}(u) .
$$

Considérons une constante positive $A$ suffisamment grande et posons

$$
T_{2}:=\max (A u|\xi|,(\log y) / 2) .
$$


On a

$$
K_{11}(u)=\frac{1}{2 i \pi} \int_{\substack{\beta=-\xi \\(\log y) / 2<|t| \leq T_{2}}} \widehat{\varrho}_{k}(v) e^{u v} d v+\frac{1}{2 i \pi} \int_{\substack{\beta=-\xi \\|t|>T_{2}}} \widehat{\varrho}_{k}(v) e^{u v} d v
$$

Lorsque $A u|\xi| \leq(\log y) / 2$, la première intégrale est nulle.

Pour estimer la seconde intégrale on utilise (3.6) car on a bien $|t|>T_{2}>$ $u|\xi|$ et on obtient

$$
\begin{aligned}
& \frac{1}{2 i \pi} \int_{\substack{\beta=-\xi \\
|t|>T_{2}}} \widehat{\varrho}_{k}(v) e^{u v} d v \\
& \quad=\frac{1}{2 i \pi}\left(\int_{\substack{\beta=-\xi \\
|t|>T_{2}}}(i t)^{-k} e^{u v} d v+O\left(u|\xi| e^{-u \xi} \int_{|t|>T_{2}}|t|^{-k-1} d t\right)\right) .
\end{aligned}
$$

La seconde formule de la moyenne implique donc

$$
\frac{1}{2 i \pi} \int_{\substack{\beta=-\xi \\|t|>T_{2}}} \widehat{\varrho}_{k}(v) e^{u v} d v \ll u|\xi| e^{-u \xi} T_{2}^{-k}
$$

Il en résulte que

$$
K_{11}(u) \ll \varrho_{k}(u)(\log y)^{-k} \quad(A u|\xi| \leq(\log y) / 2),
$$

où nous avons tenu compte de (3.8).

Lorsque $A u \xi>(\log y) / 2$, on a

$$
\int_{\substack{\beta=-\xi \\(\log y) / 2<|t| \leq T_{2}}} \widehat{\varrho}_{k}(v) e^{u v} d v \ll \exp \left(k \gamma+\sigma_{0}-u \xi-u /\left(\xi^{2}+\pi^{2}\right)\right) T_{2},
$$

où l'on a utilisé (3.7), et

$$
\int_{\substack{\beta=-\xi \\|t|>T_{2}}} \widehat{\varrho}_{k}(v) e^{u v} d v \ll e^{-u \xi} T_{2}^{1-k},
$$

où l'on a fait appel à (3.6) puisque $|t|>T_{2}>u \xi$. Il suit, grâce à (3.8),

$$
K_{11}(u) \ll \varrho_{k}(u)(\log y)^{-k} \quad(A u \xi>(\log y) / 2) .
$$

Ainsi dans tous les cas on a

$$
K_{1}(u)=\varrho_{k}(u)\left(1+O\left((\log y)^{-k}\right)\right) .
$$

Estimation de $K_{2}(u)$. Rappelons (cf. [24], théorème II.5.1) que la fonction $F(s)$ est holomorphe dans le disque $|s-1|<1$ et qu'elle y admet la 
représentation en série de Taylor

$$
F(s)=\sum_{m \geq 1} a_{m}(s-1)^{m},
$$

où

$$
a_{m} \ll_{\varepsilon, k}(1+\varepsilon)^{m} .
$$

Il résulte de (4.8) que

$$
F(s)=O(s-1) \quad(|s-1| \leq 1 / 2) .
$$

Comme pour l'estimation de $K_{1}(u)$, considérons une constante positive suffisamment grande $A$ et posons

$$
T_{3}:=\min (A u|\xi|,(\log y) / 2) .
$$

On a

$$
\begin{aligned}
K_{2}(u)= & \frac{1}{2 i \pi} \int_{\substack{\beta=-\xi \\
|t| \leq T_{3}}} \widehat{\varrho}_{k}(v) F(1+v / \log y) e^{u v} d v \\
& +\frac{1}{2 i \pi} \int_{\substack{\beta=-\xi \\
T_{3}<|t| \leq(\log y) / 2}} \widehat{\varrho}_{k}(v) F(1+v / \log y) e^{u v} d v \\
= & K_{21}(u)+K_{22}(u) \quad \text { (disons). }
\end{aligned}
$$

Lorsque $A u|\xi| \geq(\log y) / 2$, la quantité $K_{22}(u)$ est identiquement nulle, et il découle de (3.7) et de (4.9) que

$$
\begin{aligned}
K_{21}(u) \ll & \frac{e^{-u \xi}}{\log y}\left((1+|\xi|) \int_{|t| \leq \pi} \exp \left(\sigma_{0}-\frac{2 t^{2}}{\pi^{2}} \sigma_{2}\right) d t\right. \\
& \left.+\int_{\pi<|t| \leq T_{3}} \exp \left(\sigma_{0}-\frac{u}{\xi^{2}+\pi^{2}}\right) u|\xi| d t\right) \\
\ll & (1+|\xi|) \varrho_{k}(u)(\log y)^{-1},
\end{aligned}
$$

d'après (3.8). Par conséquent,

$$
K_{2}(u) \ll(1+|\xi|) \varrho_{k}(u)(\log y)^{-1} \quad(A u|\xi| \geq(\log y) / 2) .
$$

Estimons maintenant $K_{2}(u)$ sous la condition $A u|\xi|<(\log y) / 2$. On déduit facilement de (3.8) et (4.10) que

$$
K_{21}(u) \ll_{k} \frac{1}{\log y} \quad(1 \leq u \leq k) .
$$

Lorsque $u>k$, on décompose $K_{21}(u)$ suivant les domaines respectifs $0<$ $|t| \leq \pi, \pi<|t| \leq T_{3}$. Il découle de (3.8) et (4.10) que

$$
K_{21}(u) \ll(1+|\xi|) \varrho_{k}(u)(\log y)^{-1} .
$$


On a donc

$$
K_{21}(u) \ll(1+|\xi|) \varrho_{k}(u)(\log y)^{-1} \quad(A u|\xi|<(\log y) / 2) .
$$

Pour estimer $K_{22}(u)$, on développe $F(1+v / \log y)$ autour du point 1 $\xi / \log y$. On obtient

$$
F\left(1+\frac{v}{\log y}\right)=\sum_{m \geq 0} b_{m}(i t)^{m}(\log y)^{-m}
$$

avec

$$
b_{0}=F(1-\xi / \log y) \ll|\xi| / \log y, \quad b_{m} \ll 1 \quad(m \geq 1) .
$$

On a d'après (3.6)

$$
\begin{aligned}
(4.15) K_{22}(u) & =\frac{1}{2 i \pi} \int_{\substack{\beta=-\xi \\
T_{3}<|t| \leq(\log y) / 2}}\left(\frac{1}{i t}\right)^{k}\left(1+O\left(\frac{u|\xi|}{|t|}\right)\right) F\left(1+\frac{v}{\log y}\right) e^{u v} d v \\
& =N(u)+R(u),
\end{aligned}
$$

où nous avons désigné par $N(u)$ la contribution du terme principal et par $R(u)$ celle du terme d'erreur. Puisque $v \ll|\xi|+t \ll t$ pour $T_{3}<t \leq$ $(\log y) / 2$, on déduit de (4.10) que l'on a

$$
\begin{aligned}
R(u) & \ll \frac{u|\xi|}{\log y} e^{-u \xi} \int_{T_{3}<|t| \leq(\log y) / 2}|t|^{-k} d t \\
& \ll \frac{e^{-u \xi+\xi}}{\log y}\left(T_{3}^{1-k}+(\log y)^{1-k}\right) \ll \varrho_{k}(u) E_{k}(x, y),
\end{aligned}
$$

car pour $k$ fixé on a $\xi \sim \xi(u) \sim \log u$. Ce qui est bien de l'ordre requis.

De plus, on a, compte tenu de (4.13),

$$
N(u)=\frac{e^{-u \xi}}{2 \pi}\left(\sum_{m \geq 0} b_{m}(\log y)^{-m} \int_{T_{3}<|t| \leq(\log y) / 2}(i t)^{m-k} e^{i u t} d t\right) .
$$

D'après la seconde formule de la moyenne, on a

$$
\int_{T_{3}<|t| \leq(\log y) / 2}(i t)^{m-k} e^{i u t} d t \ll T_{3}^{m-k}+\left(\frac{1}{2} \log y\right)^{m-k} .
$$

En reportant cette évaluation dans (4.17) on obtient

$$
\begin{aligned}
N(u) & \ll e^{-u \xi} \sum_{m \geq 0} \frac{\left|b_{m}\right|}{(\log y)^{m}}\left(T_{3}^{m-k}+\left(\frac{\log y}{2}\right)^{m-k}\right) \\
& \ll e^{-u \xi}\left(T_{3}^{-k} \sum_{m \geq 0}\left|b_{m}\right|\left(\frac{T_{3}}{\log y}\right)^{m}+\frac{1}{(\log y)^{k}} \sum_{m \geq 0}\left|b_{m}\right| 2^{-m}\right)
\end{aligned}
$$




$$
\begin{aligned}
& \ll e^{-u \xi}\left(\left|b_{0}\right| T_{3}^{-k}+T_{3}^{-k} \sum_{m \geq 1}\left(\frac{T_{3}}{\log y}\right)^{m}+\frac{1}{(\log y)^{k}}\right) \\
& \ll e^{-u \xi}\left(\frac{|\xi|}{\log y}+\frac{1}{(\log y)^{k}}\right) \ll \varrho_{k}(u) E_{k}(x, y),
\end{aligned}
$$

où nous avons fait appel à (4.14) et à l'expression asymptotique de $\varrho_{k}(u)$.

En reportant (4.16) et (4.18) dans (4.15) on obtient

$$
K_{22}(u) \ll \varrho_{k}(u) E_{k}(x, y) .
$$

Les estimations (4.12) et (4.19) nous permettent de conclure que

$$
K_{2}(u) \ll \varrho_{k}(u) E_{k}(x, y) \quad(A u|\xi|<(\log y) / 2) .
$$

En reportant (4.7) dans (4.3) et en tenant compte de (4.11) et (4.20), on obtient (4.1).

Fin de la preuve du théorème. On se place dans le cas $k \neq 1$, car le résultat est connu pour $k=1$ (cf. (1.1)). D'après une forme effective de la formule de Perron (cf. [24], théorème II.2.2), on a uniformément pour $\sigma>0$, $T>0$

$$
\begin{aligned}
S_{k}(x, y)=\frac{1}{2 i \pi} \int_{\sigma-i T}^{\sigma+i T} \zeta(s, y)^{k} & \frac{x^{s}}{s} d s \\
& +O\left(x^{\sigma} \sum_{\substack{n \geq 1 \\
P(n) \leq y}} \frac{\tau_{k}(n)}{n^{\sigma}} \frac{1}{1+T|\log (x / n)|}\right) .
\end{aligned}
$$

Choisissons $\sigma=\alpha$ et $T:=L_{\varepsilon / 2}(y)$. Posons

$$
\begin{aligned}
& R_{1}:=\left|\frac{1}{2 i \pi} \int_{\substack{\sigma=\alpha \\
1 / 2<|t|<L_{\varepsilon / 2}(y)}} \zeta(s, y)^{k} \frac{x^{s}}{s} d s\right|, \\
& R_{2}:=x^{\alpha} \sum_{\substack{n \geq 1 \\
P(n) \leq y}} \frac{\tau_{k}(n)}{n^{\alpha}} \frac{1}{1+L_{\varepsilon / 2}(y)|\log (x / n)|} .
\end{aligned}
$$

On a donc

$$
S_{k}(x, y)=K(u)+O\left(R_{1}+R_{2}\right),
$$

où $K(u)$ désigne le membre de gauche de (4.1).

Le théorème étant trivialement vérifié pour $y$ borné et $1 \leq u \leq L_{\varepsilon}(y)$, on suppose $y \geq y_{0}(\varepsilon, k)$.

La quantité $K(u)$ ayant dejà été estimée à la proposition 4.1, le théorème sera définitivement établi lorsque nous aurons prouvé que les quantités $R_{1}$ et $R_{2}$ sont englobées par le terme d'erreur de (1.16). 
Estimation de $R_{2}$. On a

$$
\begin{aligned}
R_{2} & \ll \frac{x^{\alpha}}{\sqrt{T}} \sum_{\substack{n \geq 1 \\
P(n) \leq y}} \frac{\tau_{k}(n)}{n^{\alpha}}+x^{\alpha} \sum_{\substack{|\log (x / n)| \leq T^{-1 / 2} \\
P(n) \leq y}} \frac{\tau_{k}(n)}{n^{\alpha}} \\
& =R_{21}+R_{22} \quad \text { (disons). }
\end{aligned}
$$

D'après le lemme 3.7 on a

$$
R_{22} \ll x \varrho_{k}(u) L_{\varepsilon}(y)^{-1} .
$$

De plus

$$
R_{21} \ll x^{\alpha} \zeta(\alpha, y)^{k} T^{-1 / 2} .
$$

On déduit du lemme 3.1 que

$$
R_{21} \ll x \varrho_{k}(u) L_{\varepsilon}(y)^{-1} .
$$

Ainsi on a

$$
R_{2} \ll x \varrho_{k}(u) L_{\varepsilon}(y)^{-1} .
$$

Estimation de $R_{1}$. Sous la condition $u \leq\left(\log _{2} y\right)^{2}$, on écrit

$$
R_{1}=R_{11}+R_{12},
$$

où $R_{11}, R_{12}$ correspondent respectivement à la contribution à l'intégrale de (4.21) des domaines $1 / 2<|t|<T_{4}$ et $T_{4} \leq|t|<T$, avec

$$
T_{4}:=\exp \left(\left(\log _{2} y\right)^{3}\right) \text {. }
$$

Pour évaluer $R_{11}$ on fait appel au lemme 3.1 et à la formule (3.6) car $|t| \log y>(\log y) / 2>u|\xi|$. Il vient

$$
\zeta(s, y)^{k}=\zeta(s)^{k}\left(1+O\left(\frac{1+u|\xi|}{|t| \log y}\right)\right) .
$$

On sait de plus (cf. lemme 4.3 [23]) que, pour $k$ fixé,

$$
\xi \sim \log u \quad(u \rightarrow+\infty) .
$$

Il en résulte

$$
|\xi|(\log y)^{-1} \ll(\log T)^{-1},
$$

ce qui implique classiquement

$$
\zeta(s, y)^{k} \ll(\log (1+|t|))^{k} \quad\left(\sigma=\alpha, 1 / 2<t<T_{4}\right) .
$$

Il suit

$$
\begin{aligned}
R_{11} & =\frac{1}{2 i \pi} \int_{\substack{\sigma=\alpha \\
1 / 2<|t|<T_{4}}} \zeta(s)^{k} \frac{x^{s}}{s} d s+O\left(x^{\alpha} \frac{u|\xi|}{\log y} \int_{1 / 2<|t|<T_{4}} t^{-2}(\log (1+|t|))^{k} d t\right) \\
& =\frac{1}{2 i \pi} \int_{\substack{\sigma=\alpha \\
1 / 2<|t|<T_{4}}} \zeta(s)^{k} \frac{x^{s}}{s} d s+O\left(x \varrho_{k}(u)(\log y)^{-1}\right) .
\end{aligned}
$$


On estime l'intégrale par double intégration par parties, en tenant compte des inégalités de Cauchy

$$
\left(\frac{\zeta(s)^{k}}{s}\right)^{(j)} \ll_{j} \frac{\left(\log (1+|t|)^{k}\right)^{j+1}}{|t|} .
$$

Il vient

$$
\begin{aligned}
\frac{1}{2 i \pi} \int_{\substack{\sigma=\alpha \\
1 / 2<|t|<T_{4}}} \zeta(s)^{k} \frac{x^{s}}{s} d s= & \frac{1}{2 i \pi}\left[\frac{x^{s}}{\log x} \frac{\zeta(s)^{k}}{s}-\frac{x^{s}}{(\log x)^{2}}\left(\frac{\zeta(s)^{k}}{s}\right)^{\prime}\right]_{\alpha \pm i / 2}^{\alpha \pm i T_{4}} \\
& +\frac{1}{2 i \pi(\log x)^{2}} \int_{\substack{\sigma=\alpha \\
1 / 2<|t|<T_{4}}} x^{s}\left(\frac{\zeta(s)^{k}}{s}\right)^{\prime \prime} d s .
\end{aligned}
$$

Par conséquent,

$$
\begin{aligned}
\frac{1}{2 i \pi} \int_{\substack{\sigma=\alpha \\
1 / 2<|t|<T_{4}}} \zeta(s)^{k} \frac{x^{s}}{s} d s & \ll \frac{x^{\alpha}}{\log x}+\frac{x^{\alpha}}{(\log x)^{2}} \int_{1 / 2<|t|<T_{4}} \frac{(\log (1+|t|))^{3 k}}{|t|} d t \\
& \ll x^{\alpha} / \log x .
\end{aligned}
$$

Il en découle que

$$
R_{11} \ll x \varrho_{k}(u)(\log y)^{-1} .
$$

Pour estimer $R_{12}$ on procède comme pour le lemme III.9.5.4 de [24]. On a

$$
\begin{aligned}
& \frac{1}{2 i \pi} \int_{\substack{\sigma=\alpha \\
T_{4} \leq|t|<L_{\varepsilon / 2}(y)}} \zeta(s, y)^{k} \frac{x^{s}}{s} d s= \frac{1}{2 i \pi} \sum_{\substack{n \geq 1 \\
P(n) \leq y}} \tau_{k}(n) \int_{T_{4} \leq|t|<L_{\varepsilon / 2}(y)}\left(\frac{x}{n}\right)^{s} d s \\
& \ll x^{\alpha} \sum_{\substack{n \geq 1 \\
P(n) \leq y}} \frac{\tau_{k}(n)}{n^{\alpha}\left(1+T_{4}|\log (x / n)|\right)} \\
&=x^{\alpha} \sum_{|x-n| \leq x T_{4}^{-1 / 4}} \frac{\tau_{k}(n)}{n^{\alpha}\left(1+T_{4}|\log (x / n)|\right)} \\
&+x^{\alpha} \sum_{|x-n|>x T_{4}^{-1 / 4}} \frac{\tau_{k}(n)}{n^{\alpha}\left(1+T_{4}|\log (x / n)|\right)} .
\end{aligned}
$$

D'après le (3.2) et le choix de $T_{4}$, on obtient

$$
\begin{aligned}
x^{\alpha} \sum_{|x-n|>x T_{4}^{-1 / 4}} \frac{\tau_{k}(n)}{n^{\alpha}\left(1+T_{4}|\log (x / n)|\right)} & \ll x^{\alpha} \zeta(\alpha, y)^{k} T_{4}^{-3 / 4} \\
& \ll x \varrho_{k}(u)(\log y)^{k-2} .
\end{aligned}
$$


D'autre part, on a, grâce à l'inégalité de Cauchy-Schwarz,

$$
\begin{aligned}
x^{\alpha} \sum_{|x-n| \leq x T_{4}^{-1 / 4}} \frac{\tau_{k}(n)}{n^{\alpha}\left(1+T_{4}|\log (x / n)|\right)} & \ll \sqrt{\sum_{n \leq 2 x}\left(\tau_{k}(n)\right)^{2} \sum_{|x-n| \leq x T_{4}^{-1 / 4}} 1} \\
& \ll x(\log x)^{\left(k^{2}-1\right) / 2} T_{4}^{-1 / 8} .
\end{aligned}
$$

On sait d'après $(3.9)$ que $\varrho_{k}(u) \gg u^{-2 u}(u>1)$, il en résulte

$$
x^{\alpha} \sum_{|x-n| \leq x T_{4}^{-1 / 4}} \frac{\tau_{k}(n)}{n^{\alpha}\left(1+T_{4}|\log (x / n)|\right)} \ll x \varrho_{k}(u)(\log y)^{-1} .
$$

Il suit

$$
R_{12} \ll x \varrho_{k}(u)(\log y)^{-1} .
$$

Par conséquent,

$$
R_{1} \ll x \varrho_{k}(u)(\log y)^{-1} \quad\left(u \leq\left(\log _{2} y\right)^{2}\right) .
$$

Sous la condition $u>\left(\log _{2} y\right)^{2}$ nous procédons comme pour la démonstration du théorème III.5.9 de [24].

On a pour $s=\alpha+i t$

$$
R_{1} \ll x^{\alpha} \max _{1 / 2<|t|<T}\left|\zeta(s, y)^{k}\right| \cdot \log T .
$$

Soit $A$ une constante positive arbitrairement grande. D'après le lemme 3.1 on a

$$
\zeta(s, y)^{k} \ll(\zeta(s)(s-1))^{k}(\log y)^{k} \widehat{\varrho}_{k}((s-1) \log y) .
$$

De plus,

$$
\zeta(s) \ll|t|^{1 / 2} \quad(\operatorname{Re} s=\alpha) .
$$

Il suit, compte tenu de (3.7),

$$
\begin{aligned}
\max _{1 / 2<|t|<A u|\xi|}\left|\zeta(s, y)^{k}\right| & \ll(u|\xi|)^{3 k / 2}(\log y)^{k} \exp \left(k \gamma+\sigma_{0}-u /\left(\xi^{2}+\pi^{2}\right)\right) \\
& \ll e^{\sigma_{0}}(\log y)^{-1} .
\end{aligned}
$$

De plus, d'après (3.6) on a, pour $|t| \geq A u|\xi|$,

$$
\zeta(s, y)^{k} \ll \zeta(s)^{k}\left(1+O\left(\frac{u|\xi|}{|t| \log y}\right)\right) \ll(\log (1+|t|))^{k} \ll(\log y)^{k} .
$$

Il suit

$$
R_{1} \ll x e^{-u \xi}\left(\frac{e^{\sigma_{0}}}{\log y}+(\log y)^{k}\right) \ll x \frac{\varrho_{k}(u)}{\log y} \quad\left(u>\left(\log _{2} y\right)^{2}\right) .
$$


Ainsi dans tous les cas on a

$$
R_{1} \ll x \varrho_{k}(u)(\log y)^{-1} .
$$

En reportant (4.23) et (4.26) dans (4.22) et en tenant compte de (4.1) on obtient (1.16).

5. Démonstration du théorème 2. Nous établissons tout d'abord une proposition qui nous donne une valeur approchée de $S_{k}(x, y)$.

Proposition 5.1. Soient $0<\varepsilon<1, k>0$. Pour $x, y$ dans le domaine $\left(\mathrm{H}_{\varepsilon}\right)$, on $a$

$$
\begin{aligned}
S_{k}(x, y)=x \int_{0}^{u} z_{k}(u-v) & d\left(S_{k}\left(y^{v}\right) y^{-v}\right) \\
+ & O\left(x \varrho_{k}(u) L_{\varepsilon}(y)^{-1}\right)+O\left(x L_{\varepsilon / 2}(x)^{-1}\right) .
\end{aligned}
$$

Démonstration. Posons $T=L_{\varepsilon / 2}(y)$. D'après une formule de Perron (cf. [24], théorème II.2.2), on a

$$
S_{k}(x, y)=\frac{1}{2 i \pi} \int_{\alpha-i T}^{\alpha+i T} \zeta(s, y)^{k} \frac{x^{s}}{s} d s+O\left(x^{\alpha} \sum_{\substack{n \geq 1 \\ P(n) \leq y}} \frac{\tau_{k}(n)}{n^{\alpha}} \frac{1}{(1+T|\log (x / n)|)}\right) .
$$

D'après (4.23), il suit

$$
S_{k}(x, y)=\frac{1}{2 i \pi} \int_{\alpha-i T}^{\alpha+i T} \zeta(s, y)^{k} \frac{x^{s}}{s} d s+O\left(x \varrho_{k}(u) L_{\varepsilon}(y)^{-1}\right) .
$$

Posons

$$
P(u):=\frac{1}{2 i \pi} \int_{\substack{\operatorname{Re} s=\alpha \\|t|<T}} \zeta(s, y)^{k} \frac{x^{s}}{s} d s .
$$

D'après les lemmes 3.1 et 3.10 , on a

$$
\begin{array}{r}
\frac{\zeta(s, y)^{k}}{s}=\frac{\zeta(s)^{k}}{s}((s-1) \log y) \widehat{z}_{k}((s-1) \log y)+O\left(\frac{\zeta(s, y)^{k}}{s} T^{-1}\right) \\
(\operatorname{Re} s=\alpha,|t|<T) .
\end{array}
$$

Le terme d'erreur ci-dessus fournit à l'intégrale une contribution

$$
\ll x^{\alpha} \zeta(\alpha, y)^{k} T^{-1 / 2} \ll x \varrho_{k}(u) L_{\varepsilon}(y)^{-1} .
$$

D'autre part,

$$
\frac{\zeta(s)^{k}}{s}(s-1)=\int_{0}^{\infty} e^{-v(s-1) \log y} d\left(S_{k}\left(y^{v}\right) y^{-v}\right) \quad(\sigma>1)
$$


et le théorème de convolution implique

$$
\frac{\zeta(s)^{k}}{s}((s-1) \log y) \widehat{z}_{k}((s-1) \log y)=\widehat{B}_{k}(s) \quad(\sigma>1),
$$

avec

$$
B_{k}(t)=e^{t} \int_{0}^{\infty} z_{k}\left(\frac{t}{\log y}-v\right) d\left(S_{k}\left(y^{v}\right) y^{-v}\right)
$$

On sait d'après le théorème 2 de [17] et le lemme 1.3 .1 de [20] que

$$
z_{k}(v) \ll \begin{cases}v^{k}, & k \notin \mathbb{N}, \\ e^{-v \log v}, & k \in \mathbb{N} .\end{cases}
$$

Il en résulte, en tenant compte de (1.11), que

$$
B_{k}(t) \ll t^{2 k} e^{t} .
$$

Par conséquent, $\widehat{B}_{k}(s)$ converge absolument pour $\sigma>1$.

De plus, la fonction $S_{k}\left(y^{v}\right) y^{-v}$ est à variation bornée dans tout intervalle borné. Puisque la fonction $z_{k}(v)$ possède une seule discontinuité, de première espèce, en zéro. Il découle du théorème II.11.1 de [27] que $B_{k}(t)$ est à variation bornée sur tout intervalle borné. On peut donc appliquer le théorème d'inversion de Laplace (cf. [27], théorème II.7.3).

Il vient

$$
B_{k}(t)=\frac{1}{2 i \pi} \int_{d-i \infty}^{d+i \infty} \widehat{B}_{k}(s) e^{t s} d s \quad(d>1) .
$$

La formule (3.19) nous permet d'affirmer que la fonction $\widehat{B}_{k}(s)$ est holomorphe dans toute région sans zéro de la fonction $\zeta(s)$. On peut donc remplacer dans (5.6) la droite d'intégration par une courbe quelconque joignant $d-i \infty$ à $d+i \infty$ et entièrement contenue dans le domaine sans zéro de Vinogradov. On a donc pour $d=1+1 / \log x$,

$$
P(u)=B_{k}(\log x)-\frac{1}{2 i \pi} \int_{Z} \widehat{B}_{k}(s) x^{s} d s+O\left(x \varrho_{k}(u) L_{\varepsilon}(y)^{-1}\right),
$$

où $Z$ est le chemin d'intégration apparaissant au lemme 3.10. Par ailleurs, d'après (3.6), on a

$$
\widehat{B}_{k}(s)=\frac{\zeta(s)^{k}}{s}\left(1+O\left(\frac{y^{1-\sigma}}{|t| \log y}\right)\right)=\frac{\zeta(s)^{k}}{s}+O\left(y^{1-\sigma} \frac{(\log |t|)^{k}}{t^{2} \log y}\right),
$$

dans le domaine $\left(\mathrm{H}_{\varepsilon}\right)$.

Un calcul facile permet de voir que la contribution du terme reste à l'intégrale prise sur le chemin $Z$ est

$$
\ll x \varrho_{k}(u) L_{\varepsilon}(y)^{-1}+x L_{\varepsilon / 2}(x)^{-1} \quad \text { dans }\left(\mathrm{H}_{\varepsilon}\right) .
$$


D'autre part, la contribution de $\zeta(s)^{k} / s$ à l'intégrale sur $Z$ a été évaluée par le lemme 3.10. Il suit

$$
P(u)=B_{k}(\log x)+O\left(x \varrho_{k}(u) L_{\varepsilon}(y)^{-1}\right)+x L_{\varepsilon / 2}(x)^{-1},
$$

dans le domaine $\left(\mathrm{H}_{\varepsilon}\right)$. La proposition est ainsi établie.

Fin de la preuve du théorème 2. Plaçons nous dans le domaine $\left(\mathrm{G}_{\varepsilon}\right)$. En identifiant (5.1) et (4.1), il vient

$$
\int_{0}^{u} z_{k}(u-v) d\left(S_{k}\left(y^{v}\right) y^{-v}\right)=(\log y)^{k-1} \varrho_{k}(u)\left(1+O\left(E_{k}(x, y)\right)\right) .
$$

Il suit

$$
\varrho_{k}(u) \ll \int_{0}^{u} z_{k}(u-v) d\left(S_{k}\left(y^{v}\right) y^{-v}\right) .
$$

Le théorème 2 en découle, compte tenu de (3.21).

\section{Démonstration du théorème 3}

Lemme 6.1. Soit $k>0$. Pour $u \geq 1, \xi=\xi(u / k)$, on a

$$
\varrho_{k}(u-v) \ll \varrho_{k}(u) e^{v \xi}
$$

uniformément pour $0 \leq v \leq u$.

Dé mo n stration. La preuve est similaire à celle du corollaire III.5.8.2 de [24].

Soit $u_{0}(k)$ un réel choisi de sorte que $k \xi \leq u / 2\left(u \geq u_{0}(k)\right)$.

Lorsque $1 \leq u \leq u_{0}(k)$, la majoration (6.1) est trivialement vérifiée.

Supposons $u>u_{0}(k)$. Pour $u-k \leq v \leq u$, on a $\varrho_{k}(u-v)=O(1)$, on en déduit ainsi que de (3.8) que

$$
\varrho_{k}(u-v) \ll \varrho_{k}(u) \exp (u \xi-u / 2) \ll \varrho_{k}(u) \exp (v \xi+k \xi-u / 2),
$$

ce qui nous donne bien (6.1).

Pour établir (6.1) dans le cas $v<u-k$, on utilise l'identité

$$
\sigma_{0}-u \xi=k I(\xi)-u \xi=\int_{1}^{u / k} \xi(t) d t .
$$

Il suit, grâce à (3.8), que

$$
\begin{aligned}
\varrho_{k}(u-v) & \ll \varrho_{k}(u) \exp \left(\int_{(u-v) / k}^{u / k} \xi(t) d t\right)\left(\frac{\sigma_{2}(u)}{\sigma_{2}(u-v)}\right)^{1 / 2} \\
& \ll \varrho_{k}(u) e^{v \xi}\left(\frac{\sigma_{2}(u)}{\sigma_{2}(u-v)}\right)^{1 / 2} .
\end{aligned}
$$


D'autre part, on sait (cf. lemme 4.5, [23]) que

$$
\sigma_{2}(u)=u\left(1+O\left(\frac{1}{1+\log (u / k)}\right)\right) \quad(u>k) .
$$

Cela implique

$$
\frac{\sigma_{2}(u)}{\sigma_{2}(u-v)} \ll \frac{u}{u-v} \ll 1 \quad(u>\max (1, k), v<u-k) .
$$

Lemme 6.2. Pour $v>0$, on a

$$
\left|\varrho_{k}^{\prime}(v)\right| \ll \varrho_{k}(v)(|\xi(v)|+1) .
$$

Démonstration. La majoration est triviale pour $0<v \leq k$. Supposons $v>k$. D'après l'équation

$$
v \varrho_{k}^{\prime}(v)=(k-1) \varrho_{k}(v)-k \varrho_{k}(v-1) \quad(v>0)
$$

on a

$$
\varrho_{k}^{\prime}(v)=\varrho_{k}(v)\left(\frac{k-1}{v}-k \frac{\varrho_{k}(v-1)}{v \varrho_{k}(v)}\right) .
$$

Grâce à (6.1), on peut écrire

$$
\left|\varrho_{k}^{\prime}(v)\right| \ll \varrho_{k}(v)\left(\frac{k-1}{v}+k \frac{e^{\xi(v / k)}}{v}\right) \ll \varrho_{k}(v)(\xi(v)+1),
$$

où nous avons tenu compte de l'estimation $\xi(v) \sim \xi(v / k)$ ( $k$ fixé).

Lemme 6.3. Pour $M \geq 1,1 \leq u \leq L_{\varepsilon}(y)$, on $a$

$$
\sum_{\substack{m \leq M \\ P(m) \leq y}} \frac{|h(m)|}{m} \varrho_{k}\left(u-u_{m}\right) \ll_{\varepsilon} \varrho_{k}(u),
$$

où l'on a posé $u_{m}:=\log m / \log y$.

Démonstration. Posons

$$
H_{1}(t, y):=\sum_{\substack{m \leq t \\ P(m) \leq y}} \frac{|h(m)|}{m} \text { et } \quad C_{|h|}(y):=\sum_{\substack{m>1 \\ P(m) \leq y}} \frac{|h(m)|}{m} .
$$

Par intégration par parties, on a

$$
\begin{aligned}
& \sum_{\substack{m \leq M \\
P(m) \leq y}} \frac{|h(m)|}{m} \varrho_{k}\left(u-u_{m}\right) \\
& \quad=H_{1}(M, y) \varrho_{k}\left(u-u_{M}\right)-\varrho_{k}(u)+\int_{1}^{M} H_{1}(t, y) \frac{\varrho_{k}^{\prime}\left(u-u_{t}\right)}{t \log y} d t
\end{aligned}
$$




$$
\begin{aligned}
& \ll C_{|h|}(y) \varrho_{k}(u)+R_{\varepsilon}(M, y) \varrho_{k}\left(u-u_{M}\right)+\int_{1}^{M} R_{\varepsilon}(t, y) \frac{\left|\varrho_{k}^{\prime}\left(u-u_{t}\right)\right|}{t \log y} d t \\
& \ll C_{|h|}(y) \varrho_{k}(u),
\end{aligned}
$$

où nous avons fait appel à (1.18), (6.1) et (6.2).

Fin de la preuve du théorème 3 . Nous pouvons supposer $y \geq$ $y_{0}(\varepsilon, k)$. D'après $(1.18)$ on a

$$
\psi_{f}(x, y)=\sum_{\substack{m \leq x / 2 \\ P(m) \leq y}} h(m) S_{k}(x / m, y)+\sum_{\substack{x / 2<m \leq x \\ P(m) \leq y}} h(m) .
$$

Grâce à (1.18) et (3.9) on peut tout de suite écrire

$$
\sum_{\substack{x / 2<m<x \\ P(m) \leq y}}|h(m)| \ll x R_{\varepsilon}(x / 2, y) \ll x \varrho_{k}(u) L_{\varepsilon}(x)^{-1} .
$$

Par ailleurs, on a grâce à (1.16)

$$
\begin{aligned}
\sum_{\substack{m \leq x / 2 \\
P(m) \leq y}} h(m) S_{k}(x / m, y) & \\
= & x(\log y)^{k-1}\left(\sum_{\substack{m \leq x / 2 \\
P(m) \leq y}} \frac{h(m)}{m} \varrho_{k}\left(u-u_{m}\right)\right. \\
& \left.+O\left(\sum_{\substack{m \leq x / 2 \\
P(m) \leq y}} \frac{|h(m)|}{m} \varrho_{k}\left(u-u_{m}\right) E_{k}(x / m, y)\right)\right) \\
= & x(\log y)^{k-1}\left(\Sigma_{1}+O\left(\Sigma_{2}\right)\right) \quad \text { (disons). }
\end{aligned}
$$

Evaluons $\Sigma_{2}$. Lorsque $1 \leq u \leq 2 k$, on a

$$
E_{k}(x / m, y)=O(1) \text {. }
$$

Il suit, compte tenu de (3.9)

$$
\Sigma_{2} \ll \varrho_{k}(u)
$$

Ceci est bien de l'ordre requis.

Autrement, on a

$$
\sum_{\substack{m \leq x / 2 \\ P(m) \leq y}} \frac{|h(m)|}{m} \varrho_{k}\left(u-u_{m}\right)\left|\xi\left(u-u_{m}\right)\right|
$$




$$
\begin{aligned}
& =\sum_{\substack{m \leq x y^{-k} \\
P(m) \leq y}} \frac{|h(m)|}{m} \varrho_{k}\left(u-u_{m}\right)\left|\xi\left(u-u_{m}\right)\right| \\
& \quad+\sum_{\substack{x y^{-k}<m \leq x / 2 \\
P(m) \leq y}} \frac{|h(m)|}{m} \varrho_{k}\left(u-u_{m}\right)\left|\xi\left(u-u_{m}\right)\right| \\
& \ll|\xi| \varrho_{k}(u)+R_{\varepsilon}(\sqrt{x}, y) \ll \varrho_{k}(u)(1+|\xi|),
\end{aligned}
$$

où nous avons tenu compte de (6.3), de la croissance de $\xi(v)$ pour $v \geq 1$ et de (3.9).

Il en résulte, puisque $E_{k}(x / m, y)=\left(1+\left|\xi\left(u-u_{m}\right)\right|\right)(\log y)^{-1}+(\log y)^{-k}$,

$$
\Sigma_{2} \ll \varrho_{k}(u) E_{k}(x, y) .
$$

Evaluons $\Sigma_{1}$. On a

$$
\begin{aligned}
& \sum_{\substack{m \leq x / 2 \\
P(m) \leq y}} \frac{h(m)}{m} \varrho_{k}\left(u-u_{m}\right) \\
& \quad=\sum_{m \leq y} \frac{h(m)}{m} \varrho_{k}\left(u-u_{m}\right)+\sum_{\substack{y<m \leq x / 2 \\
P(m) \leq y}} \frac{h(m)}{m} \varrho_{k}\left(u-u_{m}\right) .
\end{aligned}
$$

La seconde somme du terme de droite est englobée par le terme d'erreur. En effet,

$$
\left|\sum_{\substack{y<m \leq x / 2 \\ P(m) \leq y}} \frac{h(m)}{m} \varrho_{k}\left(u-u_{m}\right)\right| \ll L_{\varepsilon / 2}(y)^{-1} \quad(u \leq 2 k),
$$

ce qui est bien de l'ordre requis.

Lorsque $u>2 k$ on écrit, compte tenu de (6.1) et (3.9),

$$
\begin{aligned}
& \sum_{\substack{y<m \leq x / 2 \\
P(m) \leq y}} \frac{h(m)}{m} \varrho_{k}\left(u-u_{m}\right) \\
& =\sum_{\substack{y<m \leq x y^{-k} \\
P(m) \leq y}} \frac{h(m)}{m} \varrho_{k}\left(u-u_{m}\right)+\sum_{\substack{x y^{-k}<m \leq x / 2 \\
P(m) \leq y}} \frac{h(m)}{m} \varrho_{k}\left(u-u_{m}\right) \\
& \ll \varrho_{k}(u-1) L_{\varepsilon / 2}(y)^{-1}+R_{\varepsilon}(\sqrt{x}, y) \ll \varrho_{k}(u) L_{\varepsilon}(y)^{-1} .
\end{aligned}
$$

On estime la première somme du terme de droite de (6.7) par sommation 
d'Abel (cf. [24], théorème I.0.1). On obtient

$$
\sum_{\substack{m \leq y \\ P(m) \leq y}} \frac{h(m)}{m} \varrho_{k}\left(u-u_{m}\right)=\varrho_{k}(u-1) H(y, y)+\int_{1}^{y} H(t, t) \frac{\varrho_{k}^{\prime}\left(u-u_{t}\right)}{t \log y} d t
$$

où nous avons posé

$$
H(t, z):=\sum_{\substack{m \leq t \\ P(m) \leq z}} \frac{h(m)}{m} .
$$

De plus, il est clair que

$$
H(t, t)=C_{f}+O\left(L_{\varepsilon / 2}(t)^{-1}\right) .
$$

Il suit

$$
\begin{aligned}
& \sum_{\substack{m \leq y \\
P(m) \leq y}} \frac{h(m)}{m} \varrho_{k}\left(u-u_{m}\right) \\
= & C_{f} \varrho_{k}(u)+L_{\varepsilon / 2}(y)^{-1} \varrho_{k}(u-1)+O\left(\int_{1}^{y} L_{\varepsilon / 2}(t)^{-1} \frac{\left|\varrho_{k}^{\prime}\left(u-u_{t}\right)\right|}{t \log y} d t\right) .
\end{aligned}
$$

On a donc au vu des conditions (1.18)

$$
\sum_{\substack{m \leq y \\ P(m) \leq y}} \frac{h(m)}{m} \varrho_{k}\left(u-u_{m}\right)=C_{f} \varrho_{k}(u)+O\left((1+|\xi|) \varrho_{k}(u)(\log y)^{-1}\right) .
$$

En reportant (6.8) et (6.9) dans (6.7) et puis dans (6.5) et en tenant compte de (6.6), on obtient le théorème 3 .

\section{Bibliographie}

[1] K. Alladi, Asymptotic estimates of sums involving the Moebius function. II, Trans. Amer. Math. Soc. 272 (1982), 87-105.

[2] -, An Erdös-Kac theorem for integers without large prime factors, Acta Arith. 49 (1987), 81-105.

[3] N. G. de Bruijn, On the number of positive integers $\leq x$ and free of prime factors $>y$, Nederl. Akad. Wetensch. Proc. Ser. A 54 (1951), 50-60.

[4] N. G. de Bruijn and Y. H. van Lint, Incomplete sums of multiplicative functions. I, II, ibid. 67 (1964), 339-347; 348-359.

[5] J.-M. De Koninck and D. Hensley, Sums taken over $n \leq x$ with prime factors $\leq y$ of $z^{\Omega(n)}$, and their derivatives with respect to $z$, J. Indian Math. Soc. (N.S.) $42(1978-79), 353-365$.

[6] W. J. Ellison et M. Mendès France, Les nombres premiers, Hermann, 1975.

[7] E. Fouvry et G. Tenenbaum, Diviseurs de Titchmarsh des entiers sans grand facteur premier, prépublication. 
[8] E. Fouvry et G. Tenen baum, Entiers sans grand facteur premier en progressions arithmétiques, Proc. London Math. Soc. 63 (1991), 449-494.

[9] D. G. Hazlewood, On integers all of whose prime factors are small, Bull. London Math. Soc. 5 (1973), 159-163.

[10] - Sums over positive integers with few prime factors, J. Number Theory 7 (1975), 189-207.

[11] -, Sums over numbers with restricted prime factors, ibid. 17 (1983), 350-365.

[12] D. Hensley, The convolution powers of the Dickman function, J. London Math. Soc. (2) 33 (1986), 395-406.

[13] A. Hildebrand, On the number of positive integers $\leq x$ and free of prime factors $>y$, J. Number Theory 22 (1986), 289-307.

[14] - , The asymptotic behavior of the solutions of a class of differential-difference equations, J. London Math. Soc. (2) 42 (1990), 11-31.

[15] —, On a problem of Erdös and Alladi, Monatsh. Math. 97 (1984), 119-124.

[16] A. Hildebrand and G. Tenenbaum, On integers free of large prime factors, Trans. Amer. Math. Soc. 296 (1986), 265-290.

[17] -, - , On a class of differential-difference equations arising in number theory, prépublication.

[18] A. Ivić, On squarefree numbers with restricted prime factors, Studia Sci. Math. Hungar. 20 (1985), 189-192.

[19] A. Ivić and G. Tenenbaum, Local densities over integers free of large prime factors, Quart. J. Math. Oxford Ser. (2) 37 (1986), 401-417.

[20] B. V. Levin and A. S. F ă̌nle ̌̆b, Application of some integral equations to problems in number theory, Russian Math. Surveys 22 (3) (1967), 119-204.

[21] E. Saias, Sur le nombre des entiers sans grand facteur premier, J. Number Theory 32 (1989), 78-99.

[22] A. Selberg, Note on a paper by L. G. Sathe, J. Indian Math. Soc. (N.S.) 18 (1954), $83-87$.

[23] H. Smida, Sur les puissances de convolution de la fonction de Dickman, Acta Arith. 59 (1991), 123-143.

[24] G. Tenenbaum, Introduction à la théorie analytique et probabiliste des nombres, Publ. Institut Elie Cartan 13, Univ. de Nancy 1, 1990.

[25] —, La méthode du col en théorie analytique des nombres, dans: Séminaire de Théorie des Nombres, Paris 1986-87, Progr. Math. 75, Birkhäuser, 1988, 411-441.

[26] —, Sur un problème d'Erdös et Alladi, dans : Séminaire de Théorie des Nombres, Paris 1988-1989, Progr. Math. 91, Birkhäuser, 1990, 221-239.

[27] D. T. Widder, The Laplace Transform, Princeton University Press, 1946.

[28] T. Z. Xuan, The average order of divisor functions over integers without large prime factors, Chinese Ann. Math. Ser. A 12 (1991), suppl., 28-33 (in Chinese).

[29] - The average order of $d_{k}(n)$ over integers free of large prime factors, Acta Arith. 55 (1990), 249-260.

DÉPARTEMENT DE MATHÉMATIQUES

FACULTÉ DES SCIENCES DE TUNIS

CAMPUS UNIVERSITAIRE

1060 TUNIS, TUNISIE 